\title{
"Koman i lé" : An Online Self-Reported Symptoms Surveillance System in Reunion Island
}

\author{
Nadège Marguerite ${ }^{1}$, Pascal Vilain $^{\star 1}$, Etienne Sévin $^{2}$, Farid Sahridjii ${ }^{2}$ and Laurent Filleul'
}

${ }^{1}$ French Institute for Public Health Surveillance, Saint-Denis Cedex 9, Réunion; ${ }^{2}$ Epiconcept, Paris, France

\section{Objective}

To describe a new surveillance system based on an online selfreported symptoms and to present the first results.

\section{Introduction}

During the past ten years, the syndromic surveillance has mainly developed thanks to clinical data sources (i.e. emergency department, emergency medical call system, etc.). However, in these systems, the population doesn't play an active role. It is now important that the population becomes an actor of this surveillance; especially since several European experiences about influenza showed that the population could participate to an internet-based monitoring.

In Reunion Island, the population is very sensitive to public health concerns. In this context, the health authorities implemented since April 2014 a web-based surveillance system, called "Koman i lé", that allows to follow the perceived health among people who don't systematically see their general practitioner.

\section{Methods}

The surveillance system is based on the use of the Internet-based cohort. Individual volunteers aged over 18 with internet access and living in Reunion Island are included. During the registration, sociodemographical data are collected. Upon registration, each user is sent a weekly email, taking them to the "Koman i lé" website. They fill in a short survey asking if they had any of the 17 symptoms during the previous week: fever, cough, headache, diarrhea, vomiting, nausea, stomac ache, sore throat, muscle pain, weakness, skin rash, red eyes, asthma attack, asthenia, rhinorrhea, dyspnoea, joint pain.

Indicators are constructed from syndromic groups. Each week, epidemiologists analyse the symptoms and indicators by age group, gender, occupational category and zip code; and make a report as a feedback to the participants (Figure1). Moreover, in the website a custom feedback is given to each volunteer so that they can compare their health to that of the population. In addition, it is also possible to make ad hoc surveys on various public health subjects.

\section{Results}

As of August 6, 2015, there are 359 participants from 22 of the 24 cities of the island. Among them, $67 \%$ are women, and $46 \%$ are between 30 and 44 years old. Thirty-three percent of the users live in Saint-Denis, the capital of Reunion Island. Since the beginning of "Koman i lé", the three most frequently reported symptoms are tiredness, rhinorrhea, and headache. The weekly monitoring of the different indicators highlighted an increase in the percentage of participants who presented an influenza-like illness in weeks 24 and 25 in 2014. This increased coincided with the start of an ILI outbreak detected by the other surveillance systems. Moreover, "Koman i lé" also allowed to observe an increase in participants who had red eyes during a major outbreak of conjunctivitis that occurred from January to April 2015.

\section{Conclusions}

The surveillance system allows the setting up of a volunteers' cohort in general population. The first results show that it possible from the data collected each week to monitor the health of the population and to detect unusual or expected health events and to follow an epidemic, despite the low number of participants. With a larger user base, systems like "Koman i lé" will help to improve the health surveillance on Reunion Island. In conclusion, the sentinel population project is original because for the first time the general population participates to syndromic surveillance. Information reported by individuals can increase traditional public health methods for more timely detection of disease outbreaks. Finally, the sentinel population allows the population of Reunion Island to take an active part in the health regional policy.

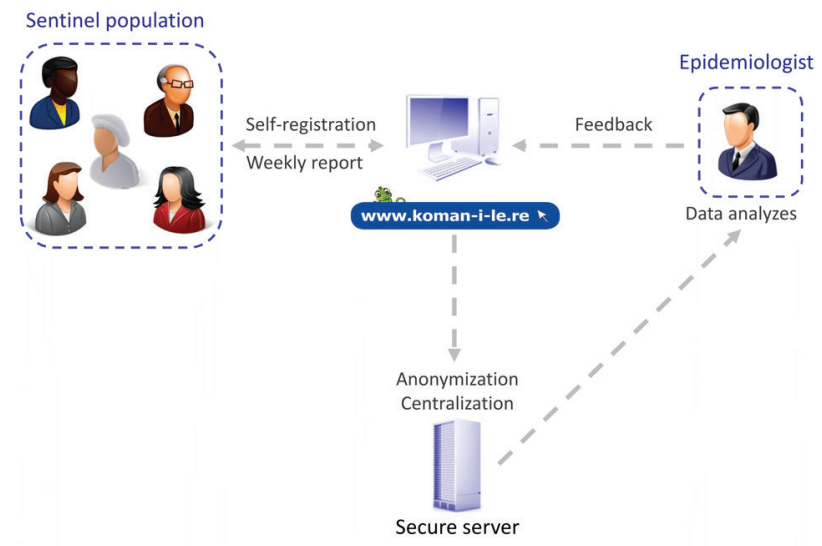

Figure1. Organization of the population sentinel project

\section{Keywords}

Sentinel population; Web-based monitoring; Syndromic surveillance

\section{Acknowledgments}

All "Koman i lé" users who have contributed information.

*Pascal Vilain

E-mail: pascal.vilain@ars.sante.fr 